\title{
Acute myeloid and chronic lymphoid leukaemias and exposure to low-level benzene among petroleum workers
}

\author{
L Rushton ${ }^{*}, 1$ A R Schnatter ${ }^{2}$, G Tang $^{3}$ and D C Glass ${ }^{4}$ \\ ${ }^{1}$ Imperial College London, Department of Epidemiology and Biostatistics, MRC-PHE Centre for Environment and Health, St Mary's \\ Campus, Norfolk Place, London W2 1PG, UK; ${ }^{2}$ ExxonMobil Biomedical Sciences, Occupational and Public Health Division, 1545 \\ US Highway 22 East, Annandale, NJ 08801 3059, USA; ${ }^{3}$ University of Pittsburgh, Graduate School of Public Health, Department of \\ Biostatistics, 130 DeSoto Street, Pittsburgh, PA 15261, USA and ${ }^{4}$ Monash University, Centre for Occupational and Environmental \\ Health, Department of Epidemiology and Preventive Medicine, Faculty of Medicine, Nursing \& Health Sciences, The Alfred \\ Hospital, 89 Commercial Road, Melbourne Victoria 3004, Australia
}

Background: High benzene exposure causes acute myeloid leukaemia (AML). Three petroleum case-control studies identified 60 cases (241 matched controls) for AML and 80 cases (345 matched controls) for chronic lymphoid leukaemia (CLL).

Methods: Cases were classified and scored regarding uncertainty by two haematologists using available diagnostic information. Blinded quantitative benzene exposure assessment used work histories and exposure measurements adjusted for era-specific circumstances. Statistical analyses included conditional logistic regression and penalised smoothing splines.

Results: Benzene exposures were much lower than previous studies. Categorical analyses showed increased ORs for AML with several exposure metrics, although patterns were unclear; neither continuous exposure metrics nor spline analyses gave increased risks. ORs were highest in terminal workers, particularly for Tanker Drivers. No relationship was found between benzene exposure and risk of CLL, although the Australian study showed increased risks in refinery workers.

Conclusion: Overall, this study does not persuasively demonstrate a risk between benzene and AML. A previously reported strong relationship between myelodysplastic syndrome (MDS) (potentially previously reported as AML) at our study's low benzene levels suggests that MDS may be the more relevant health risk for lower exposure. Higher CLL risks in refinery workers may be due to more diverse exposures than benzene alone.

Benzene is a well-known leukemogen associated with acute myeloid leukaemia (AML) particularly at high exposure (Rinsky et al, 1987; Hayes et al, 2001; IARC 2012). This paper presents detailed results for AML and chronic lymphoid leukaemia (CLL) from a pooled study of three updated case-control studies, nested within three cohort studies of male petroleum workers in Canada (Schnatter et al, 1996), the United Kingdom (Rushton and Romaniuk 1997) and Australia (Glass et al, 2003).

\section{MATERIALS AND METHODS}

The methodology has been described in detail elsewhere (Schnatter et al, 2012). In brief, new cases of lymphohaematopoietic (LH) cancers occurring through 2006 were identified using national mortality and cancer incidence registries and, additionally, for the Australian study, by self-report $(n=3)$ if confirmed by medical documentation. A random selection (with replacement) of four age-matched control subjects (five for the Australian study) per

*Correspondence: Dr L Rushton; E-mail: I.rushton@imperial.ac.uk

Received 24 July 2013; revised 12 November 2013; accepted 17 November 2013; published online 19 December 2013

(c) 2014 Cancer Research UK. All rights reserved 0007-0920/14 
case were selected that were alive and LH cancer-free at the time of their respective case diagnosis. The Canadian and UK controls were also oil-company matched.

Available diagnostic information was used by two haematopathologists to classify, using a predetermined and agreed set of criteria, the first occurrence of a relevant diagnosis according to both the International Classification of Diseases (ICD) and World Health Organization (WHO) (Jaffe et al, 2001) schemes; because the results were similar, we report on the more recent WHO scheme. The hematopathologists scored the diagnosis certainty (high, medium or low) using diagnostic term specificity, source documentation quality and agreement between source records (high: complete specification by appropriate diagnostic terms and cytogenetic information; medium: same but no cytogenetic information; and low: disagreement between source records or ambiguity).

Work histories were constructed from company records for the UK and Canadian studies and by interview (with validation by company records where possible) in the Australian study (Armstrong et al, 1996, Lewis et al, 1997, Glass et al, 2000). Country- and era-specific measured exposure data for specific jobs and tasks were used to derive shift-averaged quantitative estimates of benzene exposure concentration (parts per million (p.p.m.)) (base-estimates (BE)). These were applied to each work-history line and adjusted for differences in workplace, materials, tasks and/or environment to give a workplace exposure (WE) (p.p.m.). A job exposure certainty score (high $=3$, medium $=2$, or low $=1$ ) was allocated based on (a) job duties, (b) facility characteristics/ technology, (c) products handled, (d) job-specific BE robustness (high: all four criteria met; medium: one of b-d lacking but job duties reasonably certain; low: $>$ b-d lacking or job duties unknown). An overall career exposure certainty score was calculated by weighting job-specific WEs by the number of years spent at each level of certainty. Six exposure metrics were derived: cumulative exposure (p.p.m.-years); duration of employment (years); average exposure intensity (p.p.m.); maximum exposure intensity (p.p.m.); peak exposure (at least 1 year in job with $>3$ p.p.m. exposure for $15-60 \mathrm{~min}$ at least weekly) (Yes/No); dermal exposure (the highest job-specific relative probability of skin contact for at least a year) (none, low, medium, high).

Smoking data were collected where available from workplace records and through interview for the Australian study.

Statistical analyses included conditional logistic regression to assess the relationship between $\mathrm{LH}$ cancers relative to benzene exposure and with penalised smoothing splines (P-splines) to examine potential dose-response relationships. Odds ratios (ORs) and $95 \%$ confidence intervals (CI) were calculated relative to a background benzene concentration for benzene exposure metric tertiles, with cut-points based on exposure distributions among controls. Subgroup analyses were conducted specific to study, facility type and job. SAS version 9.2 was used for analyses (SAS Institute Inc. 2008).

Ethical approval for the combined study and recruitment of new subjects was obtained from the relevant ethics committees.

\section{RESULTS}

There were 60 cases of AML (241 controls) and 80 cases of CLL (345 controls). Mean average intensity of benzene exposure for AML cases and controls was 0.18 p.p.m. and 0.2 p.p.m., respectively, and for CLL cases and controls was 0.19 p.p.m. and 0.21 p.p.m., respectively.

Acute myeloid leukaemia. Odds ratios for AML rise as cumulative exposure, duration of exposure, peak exposure and dermal exposure increase but no clear pattern emerges for average or maximum intensity of exposure (Table 1). Sensitivity analyses by disease certainty and exposure certainty did not strengthen these results nor did restricting the exposure window to 2-20 years before case diagnosis (Supplementary Table S1).

Penalised spline curves (Figure 1) do not show a compelling dose-response relationship with continuous benzene exposure metrics, although cumulative exposure for AML hints at a possible relationship (AML $\mathrm{p}_{\text {spline }}=0.14$ ). The $\mathrm{OR}$ for $\mathrm{AML}$ associated with peak exposure is raised (Table 1), and ORs generally increase with increasing probability of dermal exposure; these patterns strengthened for Terminal Workers who are usually the highest exposed workers, for example, for peak exposure $\mathrm{OR}=1.62$, $(95 \%$ CI: $0.83,3.16$ ).

For those participants where smoking data were available, 16 out of 22 cases and 67 out of 95 controls smoked (OR: 1.5, 95\% CI: $0.44,5.14)$.

The magnitude of the ORs differed by study, with a tendency for reduced ORs for the Canadian study and increased non-significant ORs for the UK and Australian studies for all exposure metrics.

The risk of AML for ever being a tanker driver (mostly based at terminals) for at least 1 year was statistically significantly raised (OR: 2.02, 95\% CI: 1.08, 3.78) (30 cases, 82 controls) as was the risk for tanker drivers who had ever worked at a terminal (OR: 2.41, 95\% CI: 1.21, 4.80) (21 cases, 85 controls).

Chronic lymphoid leukaemia. In contrast to the results for AML, there was no increase or decrease in risk with any of the exposure metrics for CLL either as continuous variables (Supplementary Figure S1) or in categorical analyses (Table 1). Categorical analyses gave raised ORs with the highest OR for duration in tertile 2 (OR: 2.14, 95\% CI: 1.08, 4.23), although showing a downward trend. The spline analyses also suggested an association with duration of employment. Similar results were found for medium or high diagnosis certainty score with a strengthening of the relationship for duration (Tertile 2 OR: 2.36, $95 \%$ CI: $1.16,4.81)$. Analysis of those with a medium or high job confidence score showed increased ORs in all categories of the three exposure metrics with significant excesses for tertile 3 (Supplementary Table S2).

There was no relationship with continuous metrics for the Canadian and UK studies but increases in the Australian study for cumulative exposure (OR: 1.06, 95\% CI: 1.00, 1.12), maximum benzene exposure intensity (OR: $1.45,95 \%$ CI: $0.79,2.69)$ and average benzene exposure intensity (OR: 2.27, 95\% CI: 0.64, 8.02). Similar patterns were found in categorical analyses (Supplementary Table S3). However, the reference categories for the Australian study contain small numbers of cases, and there is a tendency for ORs to decrease in the last tertiles. Combining the first 2 categories of each of the metrics in Supplementary Table S3 for the Australian study greatly reduced the OR: for cumulative exposure $>2.93$ p.p.m.-years (OR: 1.75, 95\% CI: 0.71, 4.36) and reduced the ORs in all categories in the other metrics while showing the same patterns. Those who had ever worked in a refinery in the Australian study showed similar patterns of results compared with those who had never worked in a refinery (Table 2), although generally ORs were much higher in those who had ever worked in a refinery.

\section{DISCUSSION}

Overall, this study did not show a strong or consistent relationship between benzene exposure and AML or CLL. The study has high quality, precise benzene exposure estimates for individual participants, tailored to their job title, site and era and high quality diagnoses. Key components of the study analysis were replicated and confirmed by a second independent analyst. Potential limitations include small numbers in some subgroup analyses, no 
Table 1. Odds ratios and 95\% confidence intervals for acute myeloid leukaemias and chronic lymphoid leukaemias for benzene exposure metrics

Acute myeloid leukaemia

Chronic lymphoid leukaemia

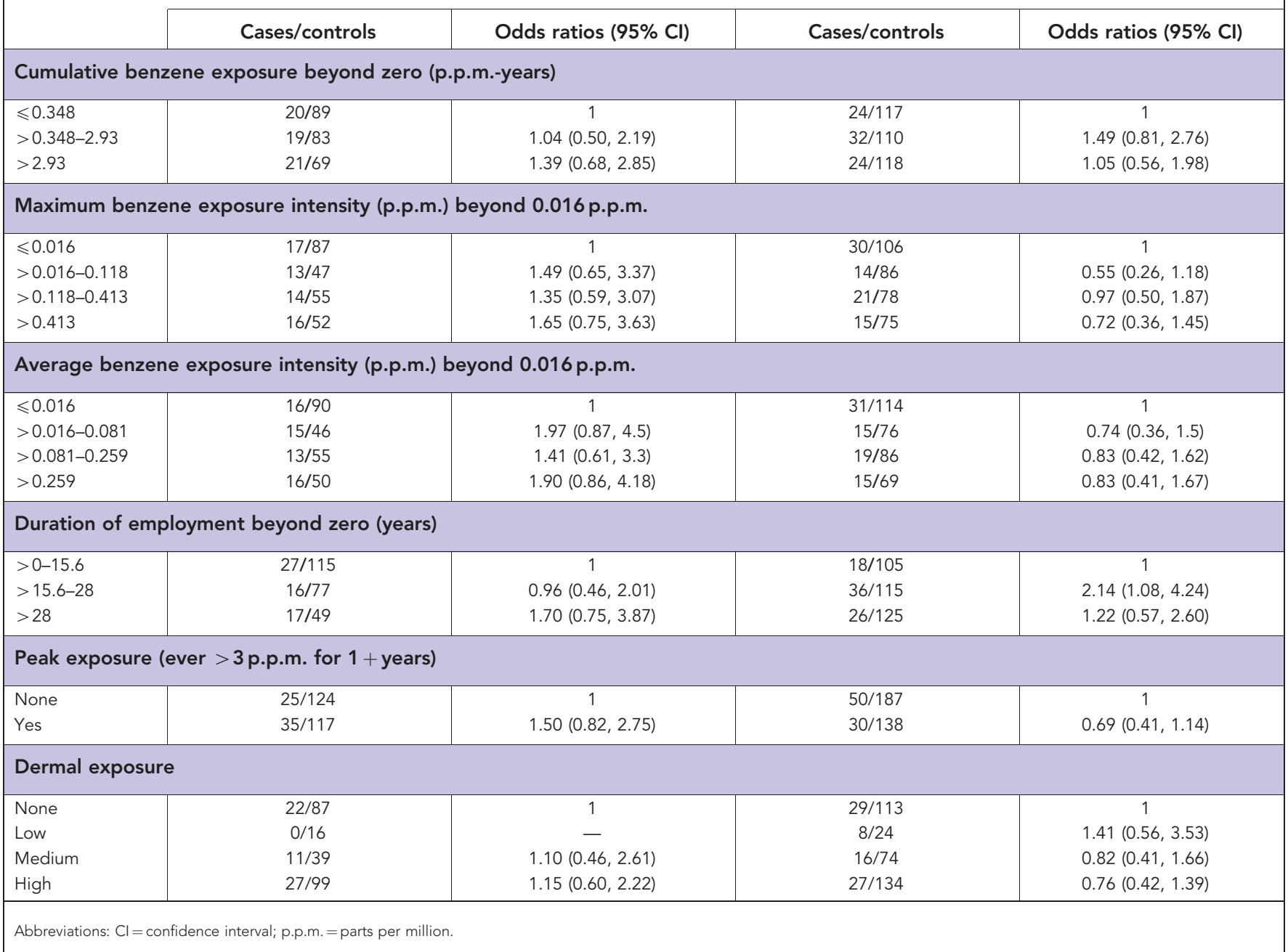

information on potential confounders, such as alcohol use, radiation exposure and genetic susceptibility, and a paucity of smoking data. Consistently elevated risks of about 1.5 for AML and smoking suggest smoking is not a potent confounder (Siegel 1993).

The lack of a strong relationship between AML and benzene exposure differed from the original studies, which had some patterns of increased risk, particularly in the Australian study (Glass et al, 2003).

A strong relationship of AML with high exposures to benzene has been shown (Crump 1994). We found lower ORs for AML than for MDS, where a strong dose-response relationship with benzene was found (Schnatter et al, 2012). This may reflect the fact that our exposures were generally low; they are similar to typical current European and North American exposures of $\leqslant 1$ p.p.m. (Wijngaarden and Stewart 2003; Capleton and Levy 2005). Our tertiles of cumulative exposure, defined as $\leqslant 0.348,0.348-2.93$, $>2.93$ p.p.m.-years, are much lower than the low cumulative exposure category of <40 p.p.m.-years in Khalade et al (2010) (meta-OR: 1.94, 95\% CI: 0.95, 3.95) and those found in studies such as the Pliofilm (Crump 1994) and Chinese occupational studies (Hayes et al, 1997; Wong et al, 2010).

Some AMLs from our earlier studies were reclassified to MDS or myeloproliferative disease (MPD) after examination of pathology information; MDS and MPD can progress to AML (Bain 1999). Before ICD-10, MDS and MPD were not available diagnostic categories and many of our cases occurred before this, particularly in the older UK study; the more recent Australian cases were more likely to have been correctly identified.

In contrast to the patterns for MDS (Schnatter et al 2012) the AML results were not consistent across the three studies, although risks for tanker drivers and in particular at terminals were similarly raised in the two diseases.

The patterns for CLL tended to be different between the three studies with the Australian study showing increased risk in several analyses and particularly for refinery work (similar to the earlier Australian nested case-control (Glass et al, 2003)). Elevated CLL mortality has previously been identified in refinery populations (Huebner et al, 2004).

CLL is considered a subtype of non-Hodgkin lymphoma (NHL), as both are lymphoid cell malignancies (Jaffe et al, 2001). A review of 68 industrial studies reported several elevated findings for lymphoid cancers (CLL, NHL, multiple myeloma) for rubber workers and cohorts exposed to multiple chemicals (Bukowski et al, 2003). Possibly a more complex environment such as a refinery compared with terminals might tend to show an increased risk for lymphoid tumours such as CLL.

\section{CONCLUSION}

While some patterns among terminal workers, who tend to have a relatively high benzene exposure, suggest a relationship 

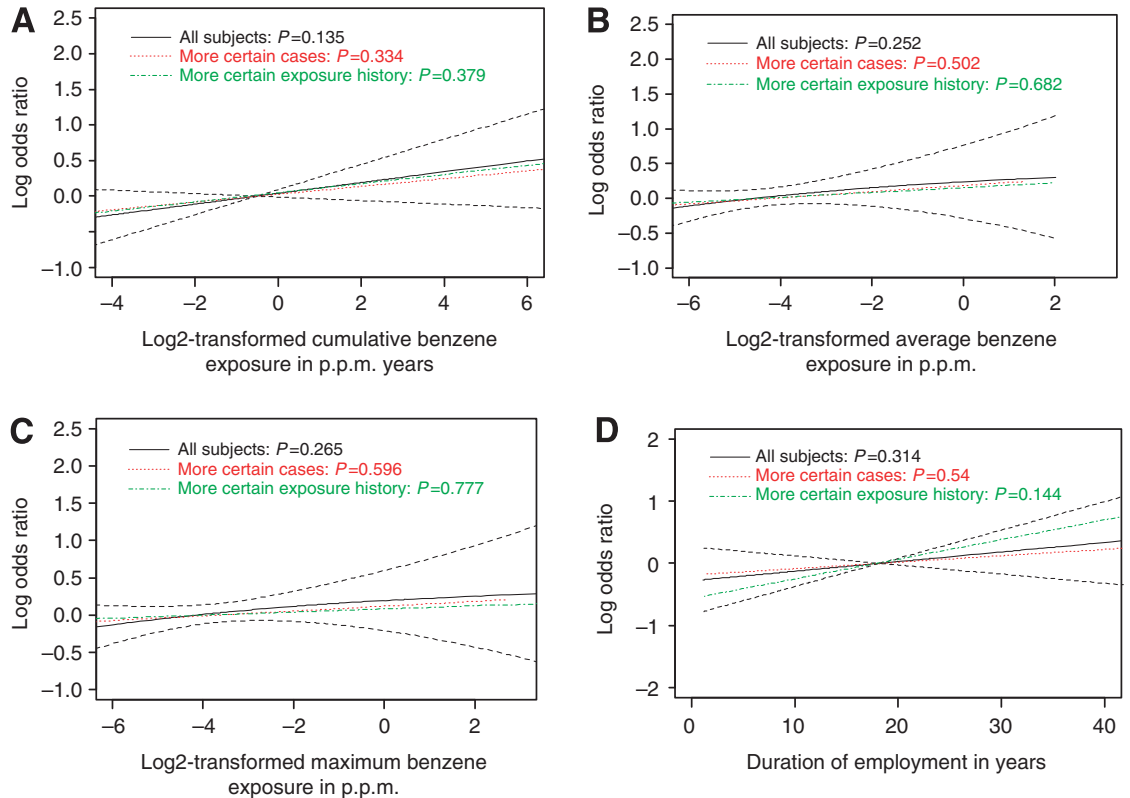

Figure 1. Penalised splines for benzene exposure metrics for AML and sensitivity analyses on disease and exposure history certainty. Log odds ratio of risk of LH cancer subtype as a smooth function of the binary logarithm of cumulative (p.p.m.-years + 0.001), average (p.p.m.), and maximum exposure (p.p.m.). In panel D, log odds ratios are plotted against duration on an additive scale. More certain cases refer to those with medium or high case certainty, and subjects with more certain exposure history refer to those with medium or high job history confidence scores. (A) AML: Cumulative benzene exposure (p.p.m.-years). (B) AML: average intensity of benzene exposure (p.p.m.). (C) AML: maximum intensity of benzene exposure (p.p.m.). (D) AML: duration of employment (years).

Table 2. Odds ratios and $95 \%$ confidence intervals for chronic lymphoid leukaemias and specific benzene exposure metrics for the Australian study ever employed at a refinery and ever not employed at a refinery

\begin{tabular}{|c|c|c|c|c|}
\hline & \multicolumn{2}{|c|}{ Ever employed at refinery } & \multicolumn{2}{|c|}{ Not ever employed at a refinery } \\
\hline & Cases/controls & Odds ratios $(95 \% \mathrm{Cl})$ & Cases/controls & Odds ratios $(95 \% \mathrm{Cl})$ \\
\hline \multicolumn{5}{|c|}{ Cumulative benzene exposure beyond zero (p.p.m.-years) } \\
\hline $\begin{array}{l}\leqslant 0.348-2.93 \\
>2.93\end{array}$ & $\begin{array}{l}3 / 15 \\
9 / 11\end{array}$ & $\begin{array}{c}1 \\
3.75(0.76,18.55)\end{array}$ & $\begin{array}{l}7 / 20 \\
4 / 13\end{array}$ & $\begin{array}{c}1 \\
0.71(0.16,3.27)\end{array}$ \\
\hline \multicolumn{5}{|c|}{ Maximum benzene exposure intensity (p.p.m.) beyond 0.016 p.p.m. } \\
\hline $\begin{array}{l}\leqslant 0.016-0.118 \\
>0.118-0.413 \\
>0.413\end{array}$ & $\begin{array}{l}2 / 13 \\
7 / 8 \\
3 / 5 \\
\end{array}$ & $\begin{array}{c}1 \\
5.65(0.87,36.83) \\
2.33(0.34,15.91)\end{array}$ & $\begin{array}{c}4 / 17 \\
3 / 8 \\
4 / 8\end{array}$ & $\begin{array}{c}1 \\
1.74(0.27,11.19) \\
1.83(0.32,10.53)\end{array}$ \\
\hline \multicolumn{5}{|c|}{ Average benzene exposure intensity (p.p.m.) beyond 0.016 p.p.m. } \\
\hline $\begin{array}{l}\leqslant 0.016-0.081 \\
>0.081-0.259 \\
>0.259\end{array}$ & $\begin{array}{c}2 / 10 \\
7 / 11 \\
3 / 5 \\
\end{array}$ & $\begin{array}{c}1 \\
2.98(0.53,16.71) \\
2.07(0.27,15.87)\end{array}$ & $\begin{array}{c}5 / 17 \\
2 / 10 \\
4 / 6 \\
\end{array}$ & $\begin{array}{c}1 \\
0.66(0.10,4.61) \\
1.67(0.32,8.70)\end{array}$ \\
\hline \multicolumn{5}{|c|}{ Duration of employment beyond zero (years) } \\
\hline $\begin{array}{l}\leqslant 15.6-28 \\
>28\end{array}$ & $\begin{array}{l}6 / 13 \\
6 / 13\end{array}$ & $\begin{array}{c}1 \\
1.14(0.10,12.66)\end{array}$ & $\begin{array}{c}9 / 28 \\
2 / 5\end{array}$ & $\begin{array}{c}1 \\
0.63(0.05,7.49)\end{array}$ \\
\hline
\end{tabular}

between AML and benzene, the overall evidence does not persuasively demonstrate a risk between benzene and AML. However, exposures in this study may have been too low to observe this. There was a general lack of association between benzene and CLL; the tendency for higher risks to occur in refinery workers may point to a role of more diverse exposures.

\section{ACKNOWLEDGEMENTS}

We thank the following for assisting with the study: John Ryder, Richard Irons, Malcolm Sim (disease classification); Tom Sorahan (UK data provision); Eileen Pearlman, Tom Armstrong, Dave Verma (exposure assessment); Susan Marcella (database 
management and programming expertise); Min Chen (statistical analyses); Elizabeth DeVilbiss (quality assurance). This work was Funded by from CONCAWE (Conservation for Clean Air and Water Europe); the American Petroleum Institute; the Aromatic Producers Association; the Canadian Petroleum Products Institute.

\section{CONFLICT OF INTEREST}

Lesley Rushton receives funding for board membership at the European Centre for Ecotoxicology and Toxicology of Chemicals and from the European Chemical Industry Council via Imperial College for project work. Rob Schnatter is employed by ExxonMobil Biomedical Sciences and has received funding from the American Petroleum Institute via the University of Colorado for studies on benzene in Shanghai, China. Deborah Glass received funding from the Australian Institute of Petroleum via Monash University for studies among petroleum workers. For the present work, funding from study sponsors to Gong Tang was received via ExxonMobil Biomedical Sciences, Inc.

\section{REFERENCES}

Armstrong TW, Pearlman ED, Schnatter AR, Bowes SM, Murray N, Nicolich MJ (1996) Retrospective benzene and total hydrocarbon exposure assessment for a petroleum marketing and distribution worker epidemiology study. Am Ind Hyg Assoc J 57: 333-343.

Bain C (1999) The relationship between the Myelodusplastic syndromes and the myeloproliferative disorders. Leukemia and Lymphoma 34(5-6): 443-449.

Bukowski JA, Huebner WW, Schnatter AR, Wojcik NC (2003) An analysis of the risk of B-lymphocyte malignancies in industrial cohorts. J Toxicol Environ Hlth 66(7): 581-597.

Capleton AC, Levy LS (2005) An overview of occupational benzene exposures and occupational exposure limits in Europe and North America. Chem Biol Interact 153-154: 43-53.

Crump K (1994) Risk of benzene-induced leukemia: a sensitivity analysis of the pliofilm cohort with additional follow-up and new exposure estimates. J Toxicol Environ Health 42: 219-242.

Glass DC, Adams GG, Manuell RW, Bisby JA (2000) Retrospective exposure assessment for benzene in the Australian Petroleum Industry. Ann Occup Hyg 44: 301-320.

Glass DC, Gray CN, Jolley DJ, Gibbons C, Sims MR, Fritschi L, Adams GG, Bisby JA, Manuell R (2003) Leukemia risk associated with low level benzene exposure. Epidemiology 15(5): 569-577.

Hayes RB, Dosemeci M, Wacholder S, Travis LB, Rothman N, Hoover RN, Linet M, Yin S-N, Li G-L, Li C-Y (1997) Benzene and the dose-related incidence of hematologic neoplasms in China. J Natl Cancer Inst 89(14): 1065-1071.

Hayes RB, Yin S-N, Dosemeci M, Linet M (2001) Benzene and Lymphohematopoetic Malignancies in Humans. Amer J Industr Med 40: 117-126.

Huebner WW, Wojcik NC, Rosamilia K, Jorgensen G, Milano CA (2004) Mortality updates (1970-1997) of two refinery/petrochemical plant cohorts at Baton Rouge, Louisiana, and Baytown, Texas. J Occup Environ Med 46(12): 1229-1245.

IARC (2012) International Agency for Research on Cancer: Monograph on the Evaluation of the Carcinogenic Risk of Chemicals, Vol 100F. WHO: Lyon.

Jaffe ES, Harris NL, Stein H, Vardiman JW (eds) (2001) World Health Organization Classification of Tumours: Pathology and Genetics of Tumours of Haematopoietic and Lymphoid Tissues. IARC Press: Lyon.

Khalade A, Jaakkola M, Pukkala E, Jaakola JJK (2010) Exposure to benzene at work and the risk of leukemia: a systematic review and meta-analysis. Environ Hlth 9: 31.

Lewis SJ, Bell GM, Cordingley N, Pearlman ED, Rushton L (1997) Retrospective estimation of exposure to benzene in a leukaemia casecontrol study of petroleum marketing and distribution workers in the United Kingdom. Occup Environ Med 54: 167-175.

Rinsky RA, Smith AB, Hornung R, Filloon TG, Young RJ, Okun AH, Landrigan PJ (1987) Benzene and leukemia: an epidemiologic risk assessment. New Eng J Med 316(17): 1044-1050.

Rushton L, Romaniuk H (1997) A case-control study to investigate the risk of leukaemia associated with exposure to benzene in petroleum marketing and distribution workers in the United Kingdom. Occup Environ Med 54: 152-166.

SAS Institute Inc. (2008) SAS/STAT 9.2 User's Guide. SAS Institute Inc: Cary, NC, USA.

Schnatter AR, Armstrong TW, Nicolich MJ, Katz AM, Huebner WH, Pearlman ED (1996) Lymphohaemotopoietic malignancies and quantitative estimates of exposure to benzene in Canadian petroleum distribution workers. Occup Environ Med 53: 773-781.

Schnatter AR, Glass DC, Tang G, Irons RD, Rushton L (2012) Myelodysplastic syndrome and benzene exposure among petroleum workers: an international pooled analysis. J Natl Cancer Inst 104(22): 1724-1737.

Siegel M (1993) Smoking and leukaemia: evaluation of a causal hypothesis. Am J Epidemiol 138: 1-9.

Wijngaarden EV, Stewart PA (2003) Critical literature review of determinants and levels of occupational benzene exposure for united states community-based case-control studies. Appl Occup Environ Hyg 18(9): 678-693.

Wong O, Harris F, Armstrong TW, Hu F (2010) A hospital-based case-control study of acute myeloid leukemia in Shanghai: analysis of environmental and occupational risk factors by subtypes of the WHO classification. Chem Biol Interact 184: 129-146.

Supplementary Information accompanies this paper on British Journal of Cancer website (http://www.nature.com/bjc) 